\title{
CADEIRA DE RODAS CONTROLADA POR SOPROS E SUCÇÕES
}

\author{
ANDRE SANCHES FONSECA SOBRINHO ${ }^{1}$ \\ KLEBER ROMERO FELIZARDO ${ }^{2}$ \\ MARCELO AUGUSTO DA SILVA ${ }^{3}$ \\ HENRIQUE PINHEIRO OLIVEIRA 4 \\ LUCAS PRADO LONE 5 \\ WALTER GERMANOVIX 6 \\ RUBERLEI GAINO ${ }^{7}$
}

FONSECA SOBRINHO, A.S; FELIZARDO, K. R.; SILVA, M. A. da; OlIVEIRA, H. P.; LONE, L. P.; GERMANOVIX, W.; GAINO, R. Cadeira de rodas controlada por sopros e sucções. Semina: Ci. Exatas/ Tecnol. Londrina, v. 21, n. 4, p. 3-7, dez. 2000.

RESUMO: Este projeto consiste na criação de uma cadeira de rodas controlada por um sistema microprocessado e acionado por sopros e sucções através de um tubo plástico, localizado a altura da boca do operador da cadeira. A aplicação direta dessa cadeira seria para o uso de pessoas tetraplégicas, e outros casos de paralisia permanente ou temporária dos membros inferiores e superiores.

PALAVRAS-CHAVE: Cadeira de rodas; LVDT; Microprocessador; Motor CC; Motor de passo.

1

O projeto em si apresenta uma alternativa de baixo custo a ser aplicada na área médica, em casos de pacientes possuidores de paralisias permanentes ou temporárias dos membros inferiores e superiores. A concepção deste tern como principal meta a locomoção do operador da cadeira de rodas, através de comandos (sopros e sucções) em um tubo localizado a altura de sua boca.

O comando succionar seria responsável pela seleção de funções básicas como ir para frente, para trás, girar para a esquerda ou direita. Assim a cada sucção executada pelo operador, uma dessas funções seria escolhida.

O comando soprar seria responsável pela execução de uma dessas funções previamente selecionada pelo comando succionar, isto é, ao realizar um sopro, a função selecionada (Ex: seguir em frente) seria executada e a cadeira começaria se deslocar para frente. Um segundo sopro interromperia a execução desta função, permitindo que se possa selecionar uma nova função e posteriormente executá-la.

Na seção 2 é descrito o projeto sobre aspectos técnicos e na seção 3 uma visão mais abrangente sobre o funcionamento.

\section{O PROJETO}

O projeto esta descriminado no conjunto de diagrama de blocos representado na Figura 1.

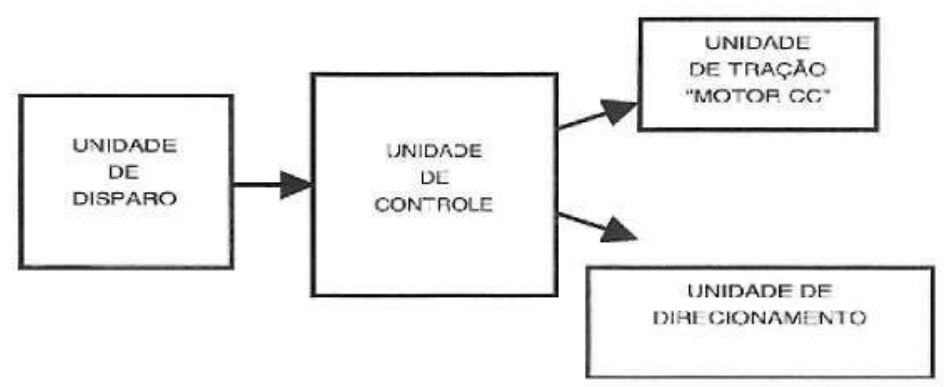

Figura 1 - Digrama de Blocos

\subsection{A Unidade de Disparo}

A Unidade de disparo tem como função gerar sinais correspondentes às ações "soprar" e "succionar", condicioná-los a forma ideal e enviálos para a unidade de controle microprocessada.

Para a construção desta unidade, utilizou-se um LVDT (Transformador Diferencial Linearmente Variável), o qual é o dispositivo responsável por gerar sinais provenientes de sopros e sucções.

1.2, 3, 4 e 5 Alunos de Graduação em Engenharia Elétrica e de Iniciação Científica.

Se 7 Docentes do Departamento de Engenharia Elétrica da Universidade Estadual de Londrina. 
Primeiramente utilizou-se o LVDT no formato convencional como mostrado na Figura 2.

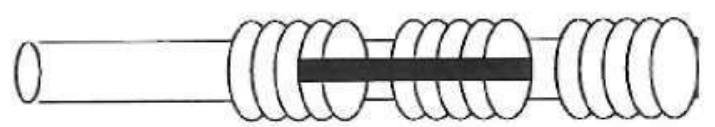

Figura 2 - LVDT simples.

Através de experimentos práticos em laboratórios observou-se o seguinte:

Houve grande perda de tensão devido as bobinas se encontrarem próximas, consequentemente as duas bobinas as quais iriam induzir o sinal de saída passaram a interferir uma na outra.

- Chegou-se a conclusão de que era necessário projetar um LVDT o qual as bobinas que induzissem o sinal de saída não estivessem muito próximas. A partir dessa idéia foi sugerido o LVDT duplo mostrado na Figura 3.

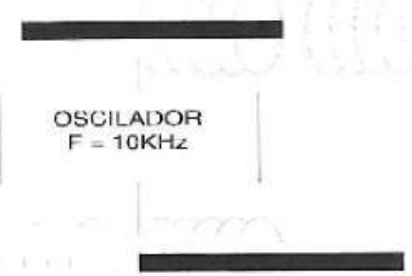

Figura 3 - LVDT duplo.

Neste formato, chega-se a seguinte idéia:

- O ramo superior do tubo só é acionado mediante a ação de soprar.

- O ramo inferior do tubo sé é acionado mediante a ação de succionar.

Além do tubo controlador, a unidade de disparo é composta também por um oscilador de amplitude $12 \mathrm{~V}$ e frequência $10 \mathrm{KHz}$ e por uma unidade de tratamento do sinal proveniente do tubo. Esta unidade de tratamento possui dois conjuntos idênticos que são ligados a cada ramo do tubo. Cada conjunto é formado por:

- Um retificador.

- Um comparador de sinais.

O diagrama de blocos da Figura 4 resume a unidade de disparo:

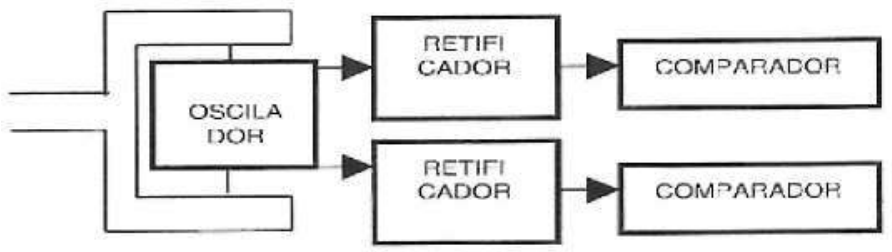

Figura 4 - Unidade de disparo.

\section{O Oscilador}

O oscilador é especificado para que a partir de um sinal DC de amplitude $12 \mathrm{~V}$ em sua entrada seja gerado um sinal $A C$ de amplitude $12 \mathrm{~V}$ com frequência de $10 \mathrm{KHz}$ em sua saída. Este sinal alimentará o sensor indutivo.

\section{O Retificador}

Trata-se de um retificador de ponte completa com filtro e offset $D C$ de $-2 \mathrm{~V}$. Tem como função retificar o sinal gerado pelo oscilador e com a ação gerar um ripple que irá para a entrada do comparador. O offset DC negativo é necessário para que só haja um sinal positivo indo para o comparador após uma intensidade de sopro razoável. Isto é um aspecto de segurança para que a cadeira não seja acionada por pequenos balanços ou trepidações.

\section{O Comparador}

É formado por amplificador operacional configurado como comparador, no qual sinais com um offset a partir de $2 \mathrm{~V}$ provenientes do retificador são saturados a 4,2V, gerando assim nível lógico " 1 " na entrada do microprocessador. Já sinais com offset menor que $2 \mathrm{~V}$ são saturados à $\mathrm{OV}$, gerando nível lógico "0".

Desta forma, ao soprar ou succionar, na saída do comparador de cada ramo é gerado um pulso.

\subsection{Unidade de Controle}

A Unidade de Controle é responsável pela coordenação das ações da cadeira de rodas, comparando os sinais de entrada e a seguir controlando as unidades de tração e de direcionamento. Esta unidade é formada por:

- Conjunto Processador (memória EPROM 2764 , processador $80 \mathrm{C} 31$, registrador 74 LS373, cristal oscilador e inversor 74LS04). 


\section{- Display Funcional.}

O diagrama de blocos da Figura 5 resume a unidade de controle.

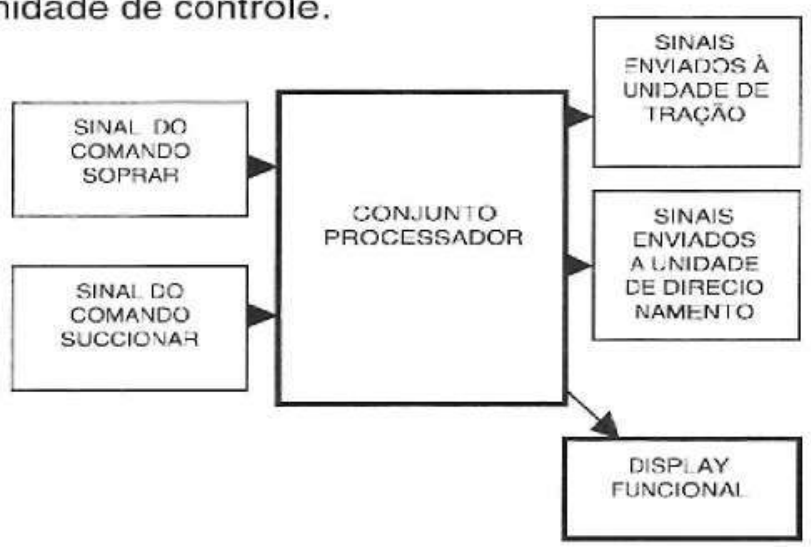

Figura 5 - Unidade de Controle.

\section{O Conjunto Processador}

O conjunto processador foi especificado da seguinte forma:

- Dois bits de entrada, um corresponde ao comando soprar e o outro ao comando succionar.

- Dez bits de saída, onde são configurados dois bits para o controle da unidade de tração, quatro bits para o controle da unidade de direcionamento e quatro bits designados para o display funcional.

\section{O Display Funcional}

O display funcional tern como função mostrar qual a função selecionada para o operador da cadeira de rodas. Possui setas indicativas que informa qual a função selecionada naquele momento (frente, trás, girar a esquerda e girar a direita). A figura 6 mostra um modelo de display funcional.

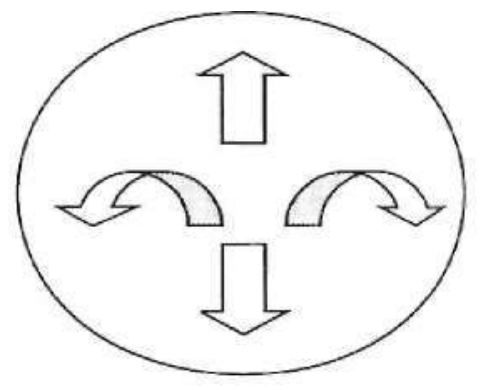

Figura 6 - Display Funcional.

\subsection{A Unidade de Direcionamento}

A Unidade de Direcionamento é comandada pelo microprocessador e formada por:

- CI ULN2003.

- Motor de Passo.
O diagrama de blocos da Figura 7 resume aunidade de direcionamento:

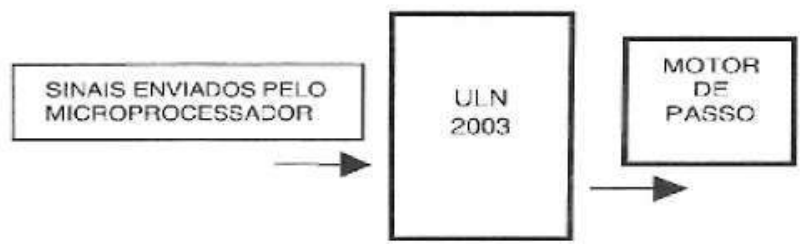

Figura 7 - Unidade de direcionamento.

\section{ULN2003}

Sua função é receber os sinais do microprocessador e enviá-los amplificados ao motor de passo para que este possa ser movimentado.

\section{Motor de Passo}

É formado por 200 passos. Possui um rotor que realiza movimentos de $60^{\circ}$ (33 passos) para a esquerda ou para a direita de acordo com o comando enviado pe!o processador (frente, trás, girar a esquerda ou direita).

\subsection{A Unidade de Tração}

A Unidade de tração é comandada pelo microprocessador e formada por:

- Estágio de Potência.

- Motor CC.

O diagrama de blocos da Figura 8 resume a unidade de tração:

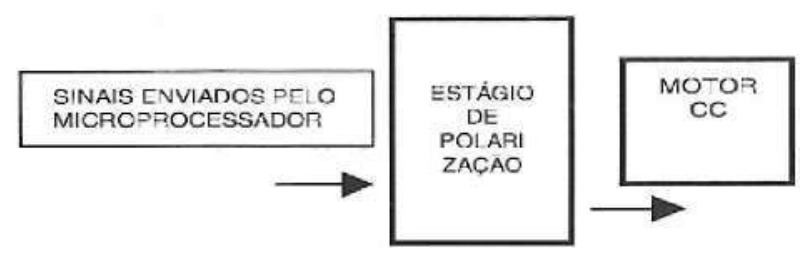

Figura 8 - Unidade de tração.

\section{Estágio de Potência}

Sua função é receber os sinais enviados pelo microprocessador e acionar o motor CC de acordo com a função escolhida. A Figura 9 mostra um estágio de potência para um motor $\mathrm{CC}(5 \mathrm{~V}, 0,5 \mathrm{~W})$

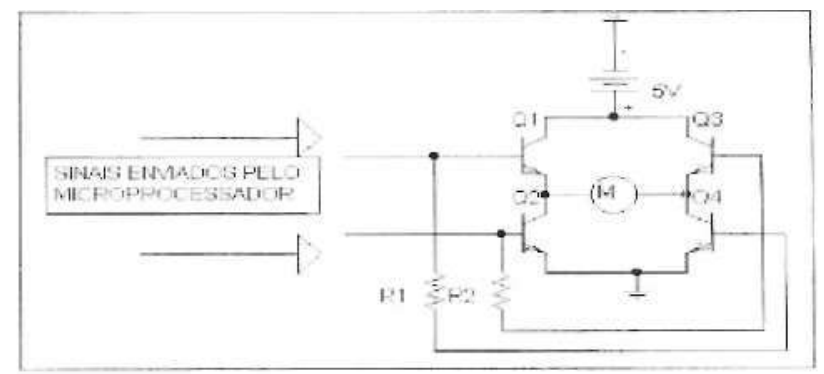

Figura 9 - Estágio de potência. 


\section{Motor CC}

O motor CC pode ser polarizado de forma a ser tracionado para frente, para trás ou permanecer imóvel.

Quando o sinal enviado pelo microprocessador chega na base de $Q 1$, este sinal e transmitido também para Q4 através de R1. Assim os transistores Q1 e Q4 ficam polarizados, fazendo com que o motor seja tracionado para frente.

Quando o sinal enviado pelo rnicroprocessador chega na base de $G 2$, este sinal é transmitido também para Q3 através de R2. Assim os transistores Q2 e Q3 tornam-se polarizados e os transistores Q1 e Q4 não. Estando Q2 e Q3 polarizados o motor é acionado para

trás.

\section{O FUNCIONAMENTO}

O procedimento a seguir visa simular o funcionamento do sistema através dos seguintes passos:

\section{1- Passo - Selecionando a função}

Através do comando succionar, seleciona-se uma função, no qual a cada sucção esta função modificada o que implica nos seguintes acontecimentos:

- Mudança no Display Funcional (Sentido Horário) que indica qual função esta selecionada no momento. Assim o display poderá estar configurado nas seguintes funções, demonstradas na Figura 10.
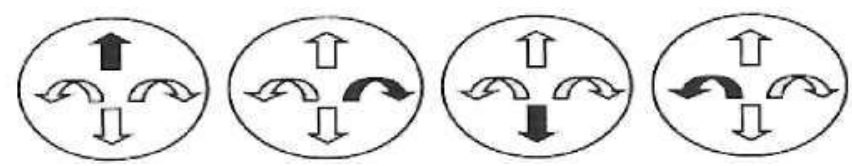

Figura 10 - Indicação de função no display: seguir em frente, girar à direita, ré e girar a esquerda.

- Mudança nos sinais enviados a Unidade de Direcionamento, que controlam a direção na qual a cadeira de rodas se movimentará. Assim a roda direcionadora presa ao motor de passo irá se mover de acordo com a função selecionada. As movimentações ocorridas na roda são demonstradas na Figura 11.
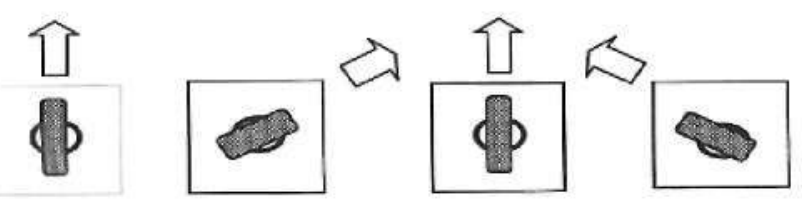

Figura 11 Posição da roda direcionadora: seguir em frente, girar à direita, ré (mesma posição de seguir em frente) e girar à esquerda.
- Mudança nos sinais que serão enviados a Unidade de Tracionamento pela Unidade de Controle Microprocessada, o qual ao ser executado - comando soprar serão configurados para realizar o tracionamento do motor CC para frente (funções "seguir em frente", "girar à direita" e "girar à esquerda") ou para trás (função "ré").

$2^{Q}$ Passo - Executando a função

Selecionada a função, basta dar um "sopro" para que a função seja executada. A seguir são mostradas as execuções para as respectivas funções:

- Seguir em Frente: A cadeira segue uma trajetória reta e para frente da posição em que se encontra.

- Girar a Direita: A cadeira segue uma trajetória curva a direita da posição em que se encontra.

- Re: A cadeira segue uma trajetória reta e para trás da posição em que se encontra.

- Girar à Esquerda: A cadeira segue uma trajetória curva a esquerda da posição em que se encontra.

A Figura 12 demonstra a movimentação da é cadeira para as funções selecionadas.

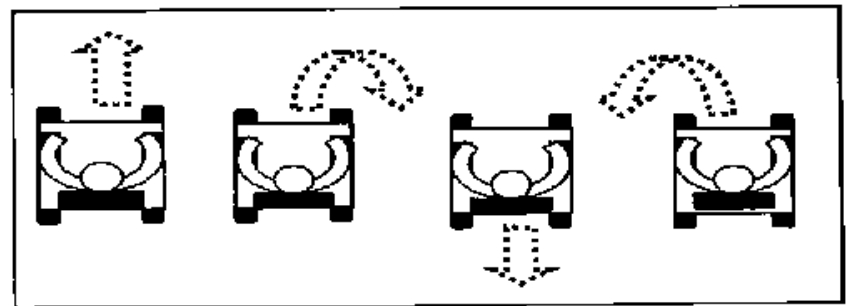

Figura 12 - Movimentação da cadeira de rodas: seguir em frente, girar à direita, ré e girar à esquerda

$3^{\text {s }}$ Passo - Interrompendo a execução de uma função

Para interromper a execução de uma função, basta um segundo sopro. Assim a movimentação da cadeira de rodas é cessada.

\section{COMENTÁRIOS FINAIS}

Em relação ao projeto inicial, houve a necessidade de promover modificações para obter-se uma melhor composição e facilidade de construção do protótipo experimental já realizado em bancada. Algumas dessas modificações já foram citadas ao longo do artigo, como a necessidade da criação de um LVDT duplo devido a interferências entre os enrolamentos.

Uma outra modificação implementada é a utilização de uma unidade de controle microprocessada. O uso do rnicroprocessador 80 C31 mostrou uma série de vantagens: 
- Melhor disposição da unidade de controle, o qual foi resumida ao conjunto processador (memória, processador, registrador, cristal e inversor) e ao display funcional. O equivalente da unidade de controle empregando circuitos integrados como registradores, contadores: biestáveis entre outros ocuparia um espaço físico muito superior, o que dificultaria ainda mais a implementação.

- Facilidade na integração da unidade de controle com as demais unidades, devido o processador apresentar uma melhor organização no envio e recepção de dados.

Maior facilidade na verificação de eventuais problemas de hardware, pois o projeto se dispõe de uma forma organizada e enxuta.
- Grande flexibilidade, o que permite mudanças (tantoem nível de hardware ou software) e inclusões de futuros dispositivos.

- Ótima relação custo-benefício, devido ao baixo custo do processador $80 \mathrm{C} 31$.

Outras modificações ainda estão em estudo, como da substituição da unidade de controle por um circuito mais simples, que não faça uso do LVDT e melhorias no estágio de potência usando um conversorCC/CC ao ser implementado o protótipo real.

A inclusão de sensores de proximidade para a segurança do usuário e a variação de velocidade de locomoção da cadeira de rodas são os próximos alvos de estudo do grupo.

FONSECA SOBRINHO, A.S; FELIZARDO, K. R.; SILVA, M. A. da; OlIVEIRA, H. P.; LONE, L. P.; GERMANOVIX, W.; GAINO, R. Wheelchair controlled by blowing or by suction. Semina: Ci. Exatas/Tecnol. Londrina, v. 21 , n. 4, p. 3-7, dez. 2000.

ABSTRACT: This project consists of the creation of a wheelchair controlled by a micro-processed system and operated by blowings and suctions through a plastic tube situated at the wheelchair's driver's mouth level. This wheelchair would be used by disabled people and other people with permanent or temporary paralysis of the lower and upper parts of the body.

KEY WORDS: wheelchair; LVDT; microprocessor; DC Motor; Step Motor.

\section{REFERÊNCIAS BIBLIOGRÁFICAS}

BOYLESTAD, Robert. Dispositivos eletronicos e teoria dos circuitos. Rio de Janeiro: Prentice-Hall do Brasil, 1998.

COBBOLD Richard S C. Transducers for biomedical measurements: principles and applications. Toronto: John Wiley \& Sons, 1974.

HELFRICK, Albert D.; COOPER, William D. Instrumentação etetronica moderna e técnicas de medição. Rio de Janeiro: Prentice-Hall do BrasiL 1994.

TANENBAUM, Andrew. Organizacao estruturada de computadores. Rio de Janeiro: Prentice-Hall do Brasil, 1992. TOMPKINS, Willis; WEBSTER, John G Interfacing sensors to the IBM PC. New Jersey: Prentice Hall, 1998. 